\title{
COMPARISON OF THE EFFICACY OF MOUTH RINSES CAMELLIA SINENSIS EXTRACT, GUAVA LEAVES EXTRACT AND SODIUM FLUO- RIDE SOLUTION, ON STREPTOCOCCUS MUTANS AND LACTOBACIL- LUS IN CHILDREN (AN IN VITRO STUDY)
}

\author{
Sara A Hassan*, Nadia E Metwalli**,Moustafa Ar Aly ${ }^{* * *}$ and Gehan G Ibrahim****
}

\begin{abstract}
Aim: The aim of the present study is to assess and compare intraorally the effectiveness of $0.5 \%$ Camellia sinensis extract, $0.5 \%$ guava leafs extract, $0.2 \%$ sodium fluoride solution on the number of Streptococcus mutans and Lactobacilli spp. in the oral cavity. Materials and Methods: Forty four healthy children of age group 7-12 years was carried out. The subjects were randomly assigned to four groups: (A) $0.5 \%$ C. sinensis extract, (B) $0.5 \%$ guava leafs extract, (C) $0.2 \%$ sodium fluoride, and (D) Saline mouth wash as control group, with 11 subjects per group. Plaque samples were taken and streptococcus mutans bacteria was assessed. Salivary samples were taken and lactobacilli bacteria was assessed. The efficacy of these extracts was tested against those bacteria through a well-diffusion method employing $10 \mathrm{ml}$ of each solution per well. Results: In the in vitro analysis Fluoride, Green tea extract and Guava extract showed significant difference between the diameters of streptococcus mutans and lactobacilli bacterial inhibition zones at different concentrations. Conclusion: In this in vitro study, there was a strong positive significant correlation between the same variables of guava extract and fluoride, but there was no significant correlation between the diameter of streptococcus mutans bacterial inhibition zones and the concentration of green tea extract, while in lactobacilli there was a strong positive significant correlation between their concentration and the diameter of bacterial inhibition zones.
\end{abstract}

\section{INTRODUCTION}

Most of mouth rinses are generally used for their analgesic, anti-microbial, anti-inflammatory, and anti-cariogenic activities. Nowadays, a wide range of mouth rinses such as chlorhexidine, sodium fluoride, and essential oils are available in market. The American Dental Association recommends that mouth rinses must be effective at modifying the micro-biota by selectively eliminating pathogens without negatively affecting the normal commensals of oral cavity ${ }^{(1)}$.

The most common plaque-mediated disease in children is dental caries, it is one of the most common chronic diseases among children. It is a preventable, localized infectious, multi-factorial disease resulting from the interaction among host, diet, and microflora on the tooth surface over a period of time, resulting in localized de-mineralization of hard tissues ${ }^{(2)}$.

The main bacterial agents in caries development are Streptococcus mutans for its initiation and Lactobacillus spp. for its progression. So, decreasing these micro-organisms causes a significant decrease in dental caries ${ }^{(3)}$.

\footnotetext{
* Post-graduate student, Department of Pediatric Dentistry and Dental Public Health, Faculty of Dentistry Ain Shams University

** Professor of Pediatric Dentistry and Dental Public Health Department, Faculty of Dentistry, Ain Shams University *** Professor of Microbiology and Immunology Department, Faculty of Medicine - Al Azhar University ***** Lecturer of Pediatric Dentistry and Dental Public Health, Faculty of Dentistry, Ain Shams University
} 
Local use of antimicrobial agents is more efficient than their systemic use, because plaque induced caries is local disease ${ }^{(4)}$.

Most of the studies demonstrated that using mouth washes in children provided a significant decrease in the decayed extracted filled surface (defs) index ${ }^{(5)}$.

Periodontal disease has been recognized as a major health problem worldwide. Periodontal diseases are infectious diseases caused by bacteria present in dental plaque. ${ }^{(6)}$ There is a direct relationship present between the presence of dental plaque and development of gingivitis ${ }^{(7)}$.

The goal of this complex periodontal treatment is to cure the inflamed tissues, by reducing the number of periodontal pathogens and alter the host response ${ }^{(8)}$.

Several studies have indicated that green tea is able to decrease the process of caries formation through several different mechanisms ${ }^{(9-10)}$.

Because Green tea is reported to be very rich in fluoride and catechin, a bioactive component, which have an anti-cariogenic efficacy by decreasing the proliferation of the streptococcal agent, interfering with the process of adhesion of the bacteria to tooth surface enamel and also act as inhibitors of glucosyltransferase and amylase ${ }^{(11)}$.

Green tea mouth wash proved to be equally effective compared to chlorhexidine which is considered as gold standard. This may also be a valuable public health intervention as it is economical and has multiple health benefits ${ }^{(12)}$.

Guava is also shown high antibacterial activity against Gram-negative and Gram-positive bacteria ${ }^{(13)}$.

Guava extract has demonstrated in vitro antiplaque actions by inhibiting growth, adherence and co-aggregation of dental plaque bacteria. Guava extracts may inhibit plaque development without disrupting homeostasis of the oral cavity. Thus, guava is an excellent antibacterial and antiplaque agent which may be a good adjunct to the mainstream periodontal treatment ${ }^{(14)}$.

Fluoride is an established antimicrobial agent. Because of its anti-cariogenic and remineralization properties, it is extensively used in the prevention of dental caries. However, due to risk of ingestion and fluoride toxicity, in addition, a high amount of systemic fluoride application is toxic ${ }^{(15)}$. So, it is not recommended in small children. Sodium fluoride is regarded as a gold standard of caries prevention.

The aim of the present study is to assess and compare intraorally the effectiveness of $0.5 \% \mathrm{Ca}$ mellia sinensis extract, $0.5 \%$ guava leafs extract, $0.2 \%$ sodium fluoride solution on the number of Streptococcus mutans and Lactobacilli spp. in the oral cavity. This study was conducted to come up with novel and cost-effective mouth washes that can be used by people for reducing the oral diseases.

\section{MATERIALS AND METHODS}

This in vitro study was conducted using three experimental groups and a control group. Randomized selection was used when groups were allocated. Totally, forty-four children from the outpatient's clinic of the Pediatric Dentistry Department at the Faculty of Dentistry, Ain Shams University, were included in this study. Their age ranged from 7-12 years old. Ethical approval was obtained from the research and ethics committee of the Faculty of Dentistry of Ain Shams University. The study was explained to all the parents' participants and they signed informed consent included their agreement to participate in this study. Also, verbal consent from the children was obtained. The children were selected according the inclusion criteria: 1- Children with no regular use of chewing xylitol gum. 2- Children without systemic disease. 3- Children without orthodontic appliance. 4- Children with healthy periodontium. 4- Children with no history of topical fluoride application. ${ }^{(16)}$, 
and its exclusion criteria: 1- Children with fixed or removable orthodontic appliances. 2- Children with prescribed antibiotics in the last month. 3- Children with gingival diseases.

Children were divided into four groups consisting of 11 child in each group. Group A: $0.5 \%$ Green tea (C. sinensis) extract. Group B: $0.5 \%$ guava leafs extract. Group C: $0.2 \%$ sodium fluoride. Group D (control group): Saline mouth wash.

\section{Preparation of mouth washes:}

Green tea mouth wash was extracted from the plant leaves of Camellia Sinensis in the laboratory of faculty of pharmacy in the pharmaceutical Department of Al-Azhar University in Cairo by an expert pharmacologist. The dried Leaves of the plant were broken into small pieces, chopped, fragmented and grounded into powdered form. The powdered net weight of the tea leaves was $2.050 \mathrm{~kg}$. Then soaked in 6 liters of $70 \%$ ethanol and were stirred 24 hours for 3 days with electrical stirrer. Preparation of the extract have been done. Thereafter, the solution was transferred into rotary flask. The rotary flask was maintained in $60^{\circ} \mathrm{c}$ temperature by the rotary evaporator (Rotavapor) in the laboratory. Then the residue of extract was scraped from the rotary flask and put in the refrigerator at $4^{\circ} \mathrm{c}$ to be used. Finally, green tea mouthwash $0.5 \%$ was prepared $(0.5 \mathrm{~g}$ of extract in $100 \mathrm{ml}$ distilled water) and poured into bottles each contains $240 \mathrm{ml}$ ( $^{(17)}$

Guava leaves extract, Guava leaves were cleaned and dried in the air for three days away from any sun rays to prevent over dryness of the leaves. The dried leaves were grounded to powdered form. The powdered net weight of the guava leaves were $2.050 \mathrm{~kg}$. Then 6 liters of $70 \%$ ethanol were added to the powder and were stirred 24 hours for 3 days with electrical stirrer. Preparation of the extract have been done. The extracts were filtered by using Whatman No. 4 filter paper and then dried in a rotary evaporator at $60^{\circ} \mathrm{C}$ in the laboratory. The dried extract was converted into powder form which was utilized for the preparation of desired concentrations of the extracts. The required concentrations of $5 \%$ of ethanolic extract was prepared by adding $0.5 \%$ of powder in $10 \mathrm{ml}$ of distilled water. The extracts was stored at $4^{\circ} \mathrm{C}$ in dark sterile bottles. ${ }^{(18)}$

Sodium fluoride mouth wash is commercially available, Fluoride mouth wash is made by ElEzaby Pharmacy by using sodium fluoride powder of Sigma Aldrich Company and adding distilled water with mannitol as a flavor. Fluoride mouth rinse is a concentrated solution for daily or weekly use. The fluoride form of mouth rinse solutions of $0.2 \%$ sodium fluoride ( $920 \mathrm{ppm}$ fluoride) were used under supervision for weekly use. ${ }^{(19)}$

Saline mouth wash (sodium chloride $0.9 \mathrm{gm} / 100 \mathrm{ml}$ ) was the control group and commercially available.

\section{Plaque samples:}

Plaque samples were taken from the outpatient clinic of the Pediatric Dentistry Department at the Faculty of Dentistry and Oral Medicine, Ain Shams University. Plaque samples were collected from each child on each experimental group using sterile buds from lingual side of lower molars, then diluted with sterile saline in a ratio of 1:100 and added to $10 \mathrm{ml}$ Thioglycolate broth to reach 1:100. ${ }^{(20)}$ The samples were transported to the laboratory of Microbiology Department of the Faculty of Medicine Al-Azhar University in ice box within 6 hours of collection. In the microbiology lab, $0.1 \mathrm{ml}$ from each dilution was spread on its selective media:

- Mitis salivarius agar (Difco Co. USA) was used as selective media for Streptococcus mutans counts.

- Tomato Juice (Rogosa) agar was used as selective media for Lactobacilli bacterial counts. ${ }^{(18)}$

Plates were incubated aerobically and anaerobically at $37^{\circ} \mathrm{C}$ for $48 \mathrm{hrs}$ using a gaspak jar then allowed to develop at room temperature for $24 \mathrm{hrs}$ to enhance colony development. All children were reinforced weekly and had oral hygiene instructions. 


\section{Saliva samples:}

Saliva samples were taken from the outpatient clinic of the Pediatric Dentistry Department at the Faculty of Dentistry and Oral Medicine, Ain Shams University. Samples were collected from each child on each experimental group by asking the child to spit in a sterile plastic container until a suitable amount of saliva was collected (in the morning before tooth brushing and breakfast). After collection of the saliva the samples were transported in ice box to the laboratory within 6 hours of collection. In the lab $0.1 \mathrm{ml}$ from each sample was spread on Tomato Juice (Rogosa) agar were used as selective media for Lactobacilli bacterial counts .Plates were incubated anaerobically at $37^{\circ} \mathrm{C}$ for 48 $96 \mathrm{hrs}$ using a gaspak jar then allowed to develop at room temperature for $24 \mathrm{hrs}$ to enhance colony development .

\section{Microbial evaluation:}

The selective medium Mitis salivarius bacitracin "MSB" (Difco Co. USA) was used as for Streptococcus mutans counts and the selective medium Tomato Juice (Rogosa) agar was used for Lactobacilli bacterial counts as following:

$1 \%$ potassium tellurite was added and then poured on sterile petri plates. After cooling to around $50 \%$, they were allowed to set for 24 hours and finally, the plaque samples were taken in serial dilutions. Then plates were incubated at around $37^{\circ} \mathrm{C}$ in the biological incubator for $24-48$ hours and clear colonies were seen on the plates, indicating the growth. The agar well-diffusion method prescribed by National Committee for Clinical Laboratory Standards (2000) were employed to analyze the antimicrobial efficacy. Suspensions of the microbial isolates were prepared in sterile normal saline and adjusted to 0.5 McFarland's standard, and were uniformly seeded by streaking sterile swab dipped in the suspension onto the Muller-Hinton agar plate surface. Wells, $5 \mathrm{~mm}$ in diameter and $4 \mathrm{~mm}$ deep, was punched on the agar plates with a sterile borer. Fifty microliters of the different mouth rinses with different concentrations were green tea extract and guava extract $0.25,0.5,1.0$ while fluoride mouth wash $0.1,0.2,0.4$ and saline as a negative control was placed in each of the wells and the plates will be incubated at $37^{\circ} \mathrm{C}$ for 24 hours . The diameter of the zone of inhibition of each mouth rinse against each microbe was measured in millimeters and recorded on the four culture plates using a Vernier caliper ${ }^{(21)}$. The plates were incubated aerobically at $37^{\circ} \mathrm{C}$ for 48-96 hours for Streptococcus mutans and anaerobically Lactobacilli for at $37^{\circ} \mathrm{C}$ for 96 hours .

\section{RESULTS}

\section{A-In vitro analysis for Streptococcus mutans:}

Intergroup comparisons of the diameters of bacterial inhibition zones (cm) of different mouth rinses:

Mean, Standard deviation (SD) values for intergroup comparisons of the diameters of bacterial inhibition zones $(\mathrm{cm})$ of different mouth rinses were presented in table (1) and figure (1)

The highest mean was recorded by Guava extract followed by Fluoride then Green tea extract while the lowest values were found in Saline. There was a significant difference between the diameters of the inhibition zones in all rinses. Pairwise comparisons showed no significant difference except for the diameters of (Saline) inhibition zones being significantly different from those of other rinses.

\section{B- In vitro analysis for Lactobacillus spp.:}

Intergroup comparisons of the diameters of bacterial inhibition zones (cm) of different mouth rinses:

Mean, Standard deviation (SD) values for intergroup comparisons of the diameters of bacterial inhibition zones $(\mathrm{cm})$ of different mouth rinses were presented in table (2) and figure (2) 
TABLE (1) Mean, Standard deviation (SD) values for intergroup comparisons of the diameters of bacterial inhibition zones $(\mathrm{cm})$ of different mouth rinses

\begin{tabular}{|c|c|c|c|c|c|}
\hline Groups & Green tea extract & Guava extract & Fluoride & Saline & P-value \\
\hline$($ mean \pm SD $)$ & $(0.72 \pm 0.53)^{\mathrm{A}}$ & $(1.07 \pm 0.63)^{\mathrm{A}}$ & $(0.80 \pm 0.54)^{\mathrm{A}}$ & $(0.00 \pm 0.00)^{\mathrm{B}}$ & $0.001 *$ \\
\hline
\end{tabular}

Different superscript letters within the same row indicates a statistically significant difference

*; significant ( $p \leq 0.05)$ ns; non-significant $(p>0.05)$

TABLE (2) Mean, Standard deviation (SD) values for intergroup comparisons of the diameters of bacterial inhibition zones $(\mathrm{cm})$ of different mouth rinses

\begin{tabular}{|c|c|c|c|c|l|}
\hline Groups & Green tea extract & Guava extract & Fluoride & Saline & P-value \\
\hline$(\mathbf{m e a n} \pm$ SD $)$ & $(0.83 \pm 0.57)^{\mathrm{A}}$ & $(0.85 \pm 0.55)^{\mathrm{A}}$ & $(0.97 \pm 0.57)^{\mathrm{A}}$ & $(0.00 \pm 0.00)^{\mathrm{B}}$ & $0.001^{*}$ \\
\hline
\end{tabular}

Different superscript letters within the same row indicates a statistically significant difference

*; significant $(p \leq 0.05) n s ;$ non-significant $(p>0.05)$

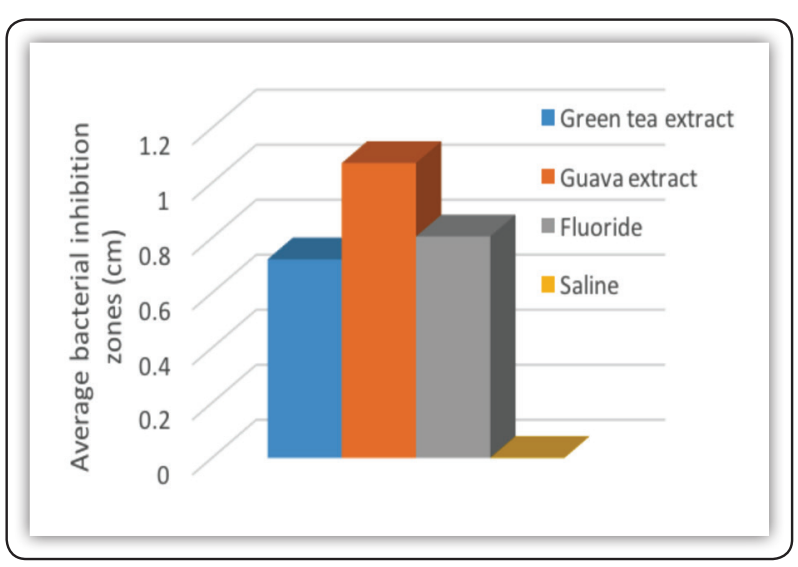

FIG (1) Bar chart showing average intergroup diameters of bacterial inhibition zones $(\mathrm{cm})$ of different mouth rinses

The highest mean was recorded by Fluoride followed by Guava extract then Green tea extract while the lowest values were found in Saline. There was a significant difference between the diameters of the inhibition zones in all rinses. Pairwise comparisons showed no significant difference except for the diameters of (Saline) inhibition zones being significantly different from those of other rinses.

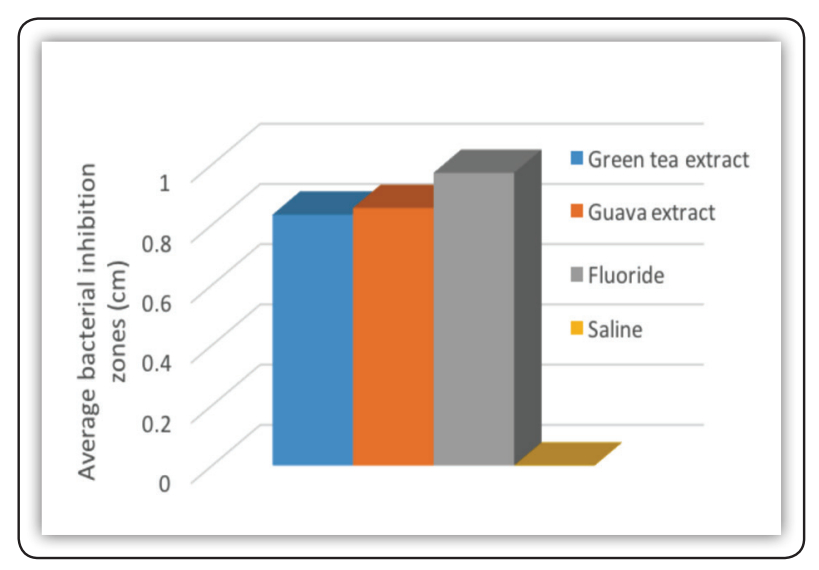

FIG (2) Bar chart showing average intergroup diameters of bacterial inhibition zones $(\mathrm{cm})$ of different mouth rinses

\section{DISCUSSION}

Dental caries is one of the most common chronic diseases in the world. It is an infectious disease caused by the colonization of bacteria ${ }^{(22)}$. Although great efforts have been made to decrease dental caries, its prevalence is still high.

As a supplementary tool alongside to mechanical methods, mouth rinses have an important role 
in the reduction of bacterial counts in the mouth, including Streptococcus mutans in decreasing dental caries ${ }^{(23-24)}$.

The aim of this study was to assess and compare intraorally the effectiveness of two different extracts, $0.5 \%$ camellia sinesis (green tea), and $0.5 \%$ guava leaves extract, with $0.2 \%$ sodium fluoride mouth washes on the bacterial count of streptococcus mutans and lactobacilli in plaque, in children ranging from 7-12 years old.

This study was conducted on children from 7-12 years old to avoid the swallowing of the mouth wash during rinsing and the toxicity of fluoride in children less than 6 years. Because high amount of fluoride ingestion may lead to acute poisoning and its low repeated ingestion causes fluorosis especially in children. So sodium fluoride mouth rinse is not recommended for children younger than 6 years ${ }^{(25)}$.

From the exclusion criteria of our study the children, not having any serious general systemic disease, they must not have taken any oral antibiotics within the last month, to avoid any factors that may affect the bacteria.

Plaque samples were chosen in this study to determine the level of streptococcus mutans and lactobacilli to eliminate the difficulties associated with collection of saliva in children. Plaque sample were taken by sterile cotton swab from the gingival $1 / 3$ of the lingual part of the $1^{\text {st }}$ molar, where plaque is likely to accumulate the most, and the brushing in this part is misused ${ }^{(26)}$.

The most commonly used mouth rinse in children for therapeutic purposes, is $0.05 \%$ sodium fluoride for daily used, but in this study we used $0.2 \%$ for weekly used ${ }^{(27)}$. As an antimicrobial agent; because of its anticariogenic and remineralization properties, it is extensively used in prevention of dental caries. But due to risk of ingestion and fluoride toxicity, it is not recommended in small children ${ }^{(28)}$.
That's why we used herbal mouth rinses that have received special attention nowadays because of being non chemical, non-synthetic, in comparison to fluoride and they have been long used in traditional medicine ${ }^{(29)}$.

In this study during preparation of green tea extract and guava extract we used $70 \%$ of ethanol alcohol as solvent system due to its effectiveness in the extraction of bioactive molecules of the plants and its safety. And electrical stirrer was used for its accuracy and stability ${ }^{(30)}$.

In the current study saline mouth wash was used as control group due to its very limited effect on bacteria and it was used because it's rinsing effect comparing to other groups.

In this study Mitis Salivarius Agar was used as a selective medium for streptococcus mutans with $1 \%$ potassium tellurite which is highly selective medium, which enable to isolate streptococci from highly contaminated specimens, as it inhibits a wide variety of bacteria. And Tomato juice was included in media for lactobacilli and was found to be advantageous for its growth, and for the isolation, cultivation and enumeration of Lactobacilli, from clinical specimens and foodstuffs ${ }^{(31)}$.

According the data of this present study there was no growth of lactobacilli before and after using the mouth rinses that were used in this study in plaque samples and that was consistent with the conducted study done by Mutisuki et al ${ }^{(32)}$. The most suitable method to detect the Lactobacillus spp. level in the oral cavity is the stimulated whole saliva method.

In this current study the in-vitro analysis showed significant inhibition zone of streptococcus mutans bacterial diameter after using green tea extract and guava extract mouth washes in different concentration which included $1 \%, 0.5 \%$, and $0.25 \%$. That showed significant different between the diameter of zone at $0.25 \%$ and $1 \%$ concentration, while the diameter at $0.5 \%$ had no significant 
difference from those of other concentrations. And this was in agreement with Singh et al $^{(33)}$ that showed the inhibitory activity of the alcoholic extract from guava leafs extract against bacteria.

There was a significant difference between the diameters of streptococcus mutans bacterial inhibition zones at different concentration of sodium fluoride mouth wash $(0.4 \%-0.2 \%-0.1 \%)$.

Regarding the lactobacilli, in-vitro analysis showed significant inhibition zone of lactobacilli bacterial diameter after using green tea extract and guava extract mouth washes in different concentration which included $1 \%, 0.5 \%$, and $0.25 \%$. Which showed significant different between the diameter of zone at $0.25 \%$ and $1 \%$ concentration, while the diameter at $0.5 \%$ had no significant difference from those of other concentrations and that was consistent with the conducted study done by Gurnani et $\mathbf{a l}^{(34)}$.

Also in this study there was a significant difference between the diameters of lactobacilli bacterial inhibition zones at different concentration of sodium fluoride mouth wash $(0.4 \%-0.2 \%-0.1 \%)$.

In this current study there was considerable inhibitory effect against S. mutans and lactobacilli spp., also there was significant difference found between the mean zones of inhibition of the different mouth rinses for the two microbes, this was agreement with Thomas et al ${ }^{(35)}$.

\section{REFERENCES}

1. John NR, Gala VC, Sawant CS.: Inhibitory effects of plant extracts on multi-species dental biofilm formation in-vitro. Int J Pharm Bio Sci. 2013; 4:487-95.

2. Tanner AC, Mathney JM, Kent RL, Chalmers NI, Hughes CV, Loo CY : Cultivable anaerobic microbiota of severe early childhood caries. J Clin Microbiol. 2011; 49:1464-74.

3. Zafar S, Harnekar SY, Siddiqi A.: Early childhood caries: Etiology, clinical considerations, consequences and management. Int Dent Saudi Arabia 2009; 11:24-36.
4. Kocak MM, Ozcan S, Kocak S, Topuz O, Erten H.: Comparison of the efficacy of three different mouth rinse solutions in decreasing the level of Streptococcus mutans in saliva. Eur J Dent 2009; 3:57-61.

5. Lobo PLD, de Carvalho CB, Fonseca SG, de Castro RS, Monteiro AJ, Fonteles MC: Sodium fluoride and chlorhexidine effect in the inhibition of Mutans Streptococci in children with dental caries: A randomized, double-blind clinical trial. Oral Microbiol Immunol. 2008; 23:486-91.

6. Kornman KS.: Mapping the pathogenesis of periodontitis: A new look. J Periodontol. 2008; 79:1560-8.

7. Powell RN: The relationship of forming and mature dental plaque to the tooth surface. J Dent Res.1965; 44:1171-5.

8. Kumar P, Ansari SH, Ali J.: Herbal remedies for the treatment of periodontal disease: A patent review. Recent Pat Drug Deliv Formul. 2009; 3:221-8.

9. Hamilton-Miller JM.: Anti-cariogenic effects of tea (Camellia sinensis), J Med Microbiol: 2001; 50:299-302.

10. Hassani AS, Amirmozafari N, Ordouzadeh N, Hamdi K, Nazari R, Ghaemi A.: Volatile components of Camellia sinensis inhibit growth and biofilm formation of oral Streptococci in vitro. Pak J Biol Sci.2008; 11:1336-41.

11. Hamilton-Miller JM.: Anti-cariogenic properties of tea (Camellia sinensis). J Med Microbiol 2001; 50:299-302.

12. Rosy S N, Srinivas R, Vikram S B, Sandhya S Y, Chandra S T, and Siva K P. : Effects of Green Tea on Streptococcus mutans Counts- A Randomised Control Trail, J. Clin Diagn Res. 2014 Nov; 8(11): 128-130).

13. Ushimaru PI, Silva MT, Di Stasi LC, Barbosa L, Fernandes A.: Antibacterial activity of medicinal plant extracts. Braz J Microbiol.2007; 38:717-9.

14. Kocak MM, Ozcan S, Kocak S, Topuz O, Erten H.: Comparison of the efficacy of three different mouth rinse solutions in decreasing the level of Streptococcus mutans in saliva. Eur J Dent 2009; 3:57-61.

15. Vargas CM.: Fluoride Supplements Prevent Caries but can Cause Mild to Moderate Fluorosis. J Evid Based Dent Pract. 2011; 11:18-20.

16. Chaturvedi TP.: Uses of turmeric in dentistry: An update. Indian J Dent Res.2009; 20:107-9.

17. Niloofar J., Moghadamnia A. A., Elaheh K., Poorsattar B. M.: The effect of Camellia Sinensis (green tea) mouthwash on plaque-induced gingivitis: a single-blinded randomized controlled clinical trial. Daru J. of Pharmaceutical Medicine. 2012; 20(1): 39. 
18. Deepika J, Pralhad D, Sandesh N, Sandeep K, Bhuvnesh A and Shilpa W.: In vitro activity of ethanolic and water extract of guava leaves at various concentrations against Lactobacillus acidophilus. J Indian ass.pub. Health dens. $2014 ; 12 ; 232-236$.

19. Steinmann J., Buer J., Pietschmann T., Steinmann E.: Antiinfective properties of epigallocatechin-3-gallate (EGCG), a component of green tea. Brit. J of Pharma.2013; Vol. 168 Issue 5, 1043-1289.

20. Neturi R., Srinivas R., Simha B., Sandhya Y., Chandra T., Siva P.: Effects of Green Tea on Streptococcus Mutans Counts- A Randomised Control Trail. J. of Clinical and Diagnostic Research : JCDR. 2014; 8:11.

21. Merritt J, Qi F.: The mutacins of Streptococcus mutans: regulation and ecology. Mol Oral Microbiol. 2012; 27(2): 57-69.

22. Jazaeri M., Rezaei S.L., Abdolsamadi H., Rafieian N.: Cariostatic Effect of Green Tea in Comparison with Common Anticariogenic Agents: An in Vitro Study. J. of D.D.D.2015; 9 (1).

23. Dean JA, Avery DR, Mc Donald RE.: Dentistry for the Child and Adolescent, 9th ed. London: Mosby Co. $2011 ; 177$.

24. Goldstep F.: Proactive intervention dentistry: a model for oral care through life. Compend. Contin. Educ. Dent. 2012; 33:394-6.

25. Vargas C.M.: Fluoride Supplements Prevent Caries but can Cause Mild to Moderate Fluorosis. J. Evid. Based Dent. Pract. 2011; 11:18-20.

26. Neturi R., Srinivas R, Simha B, Sandhya Y, Chandra T and Siva P. :Effects of Green Tea on Streptococcus Mutans Counts- A Randomised Control Trail. J. of Clinical and Diagnostic Research : JCDR. 2014; 8.11.
27. Aminabadi N. A., Balaei E., Pouralibaba F.: The Effect of $0.2 \%$ Sodium Fluoride Mouthwash in Prevention of Dental Caries According to the DMFT Index. J.O.D.D.D. 2007; 1: 2

28. Tehrani MH., Asghari G., Hajiahmadu M.: Comparing Streptococcus mutans and Lactobacillus colony count changes following green tea mouth rinse or sodium fluoride mouth rinse use in children. Dent. Res. J. 2011; 8:S58-S63.

29. Cabrera C., Artacho R., Gimenez R.: Beneficial effects of green tea - a review. Journal of American College of $\mathrm{Nu}-$ trition. 2006; 25(2):79-99.

30. Arya V., Thakur NM. , Kashyap C.: Preliminary Phytochemical Analysis of the Extracts of Psidium Leaves. J Pharmacogn Phytochem. 2012; 1: 1-5.

31. MacFaddin J. F.: Media for Isolation-Cultivation-Identification-Maintenance of Medical Bacteria. 1985; 1.

32. Motisuki C. , Monti L.L., Spolidorio P.M.D., and Pinto L.S.: Influence of sample type and collection method on Streptococcus mutans and Lactobacillus spp. counts in the oral cavity. Oral Biology. 2005; 50(3):341-345

33. Singh T., Kumar V.R., Gholap A. D., Sanap G., Anjaiah B., Roja K.: In vitro antipyrial activity of psidium guajava leaf extract. European journal of pharmaceutical and medical research. 2016; 3(1), 312-315

34. Gurnani P, Krishnan CGA, Gurnani R, Ghosh A, Shah A.: Antibacterial Activity of Guava Leaves Extract Against Lactobacillus Acidophilus: An In-Vitro Study. Int J Oral Health Med Res. 2016; 2(6):37-40

35. Thomas A., Thakur S., and Mhambrey S.: Comparison of the antimicrobial efficacy of chlorhexidine, sodium fluoride, fluoride with essential oils, alum, green tea, and garlic with lime mouth rinses on cariogenic microbes. J. Int Soc. Prev. Community Dent. 2015; 5(4): 302-308. 